\title{
CORRECTION
}

View Article Online

View Journal | View Issue

Check for updates

Cite this: J. Mater. Chem. C, 2018, 6, 4328

DOI: $10.1039 / c 8 t c 90063 a$

rsc.li/materials-c

\section{Correction: An overview of metamaterials and their achievements in wireless power transfer}

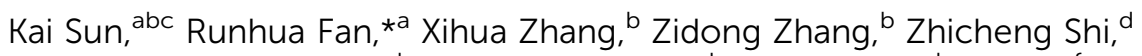 \\ Ning Wang, ${ }^{\text {e }}$ Peitao Xie, ${ }^{\mathrm{b}}$ Zhongyang Wang, ${ }^{\mathrm{b}}$ Guohua Fan, ${ }^{\mathrm{b}}$ Hu Liu, ${ }^{f}$ Chuntai Liu, ${ }^{\star}{ }^{\mathrm{f}}$ \\ Tingxi Li, ${ }^{9}$ Chao Yan $^{\mathrm{h}}$ and Zhanhu Guo*C
}

Correction for 'An overview of metamaterials and their achievements in wireless power transfer' by Kai Sun et al., J. Mater. Chem. C, 2018, DOI: 10.1039/c7tc03384b.

The authors regret that the incorrect affiliation was used for one of the authors, Ning Wang, on the original manuscript. The corrected list of authors and affiliations for this paper is shown above.

The author biography of Ning Wang was also incorrect. The corrected Biography is shown below.

Dr Ning Wang, currently a Professor at the State Key Laboratory of Marine Resource Utilization in South China Sea, Hainan University, China, obtained a PhD degree in Materials Science and Engineering from Tsinghua University, China (2007) and received two-year (2008-2010) postdoctoral training at the Department of Applied Chemistry at Nagoya University, Japan. Dr Wang is currently an executive deputy director of the State Key Laboratory of Marine Resource Utilization in South China Sea, Hainan University. His current research focuses on the fundamental science of nanomaterials applied in energy-related materials and devices.

The Royal Society of Chemistry apologises for these errors and any consequent inconvenience to authors and readers.

\footnotetext{
${ }^{a}$ College of Ocean Science and Engineering, Shanghai Maritime University, Shanghai 201306, China. E-mail: rhfan@shmtu.edu.cn

${ }^{b}$ Key Laboratory for Liquid-Solid Structural Evolution and Processing of Materials (Ministry of Education), Shandong University, Jinan, 250061, China

${ }^{c}$ Integrated Composites Laboratory (ICL), Department of Chemical \& Biomolecular Engineering, University of Tennessee, Knoxville, TN 37996, USA.

E-mail: zguo10@utk.edu

${ }^{d}$ Institute of Material Science and Engineering, Ocean University of China, Qingdao, 266100, China

${ }^{e}$ State Key Laboratory of Marine Resource Utilization in South China Sea, Hainan University, Haikou 570228, P. R. China

${ }^{f}$ National Engineering and Research Center for Advanced Polymer Processing Technology, Zhengzhou University, Zhengzhou, 450001, China

${ }^{g}$ College of Materials Science and Engineering, Shandong University of Science and Technology, Qingdao, 266590, China

${ }^{h}$ School of Material Science and Engineering, Jiangsu University of Science and Technology, Zhenjiang, Jiangsu, China
} 